\title{
The Relationship between Autopoiesis Theory and Biosemiotics: On Philosophical Suppositions as Bases for a New Information Theory
}

\author{
Yohei Nishida
}

nishida.yohei@gmail.com, Graduate School of Interdisciplinary Information Studies, University of Tokyo

\begin{abstract}
This paper discusses methodological issues related to a possible framework for a unified theory of information. We concentrate on the relationship between systems theory and semiotics, or to put it more concretely, the relationship between autopoiesis theory and biosemiotics. These theories give rise to two decisive viewpoints on life that seem potentially contradictory and consequently provoke a fruitful controversy, which is conducive for the consideration of philosophical suppositions vital for a new information theory. The following three points are derived in the context of basic principles: epistemology rather than ontology, constructivism rather than metaphysics, meta-theoretical recursiveness rather than linear consistency.
\end{abstract}

Keywords: unified theory of Information, systems theory, autopoiesis theory, semiotics, biosemiotics, Cybersemiotics, Fundamental Informatics, views of life, epistemology, constructivism, meta-theoretical recursiveness

Toward a new fundamental theory of information, this paper discusses the relationship between autopoiesis theory and biosemiotics, or to put it in a broader way, the relationship between systems theory and semiotics. As the first step in our analysis, we will also examine the cybersemiotics of Søren Brier and Fundamental Informatics of Toru Nishigaki. We are here concerned with methodological issues related to a possible framework for a unified theory of information. The question we would like to ask is what kind of philosophical suppositions are important as bases for a new information theory.

\section{Introduction}

As the development of information and communication technologies penetrates our daily lives widely and deeply, there has been growing concern with developing a new information theory. Claude Shannon's information theory, in spite of its popularity as a fundamental theory of information, is just a theory of signal transmission between telecommunication devices based on a preestablished code. It is obvious that Shannon's theory has limitations as a general theory of information because it has no direct relevance to aspects of the meaning of information.

As often pointed out, the problem with classical computational, formalistic, or mechanistic approaches to information is that they fail to cover aspects of meaning. It is for this reason that several studies have been conducted in order to create a new fundamental theory of information over the past few decades. Those discussions are concerned with various fields including phenomenology, hermeneutics, cognitive science, linguistics, semiotics, communication studies, media studies, systems theory and biology. Although it seems almost impossible to take into account all these separate fields towards a framework for a new information theory without losing overall coherence, we should not overlook the fact that there appear to be certain potential directions, such as phenomenological, semantical and biological directions. In particular, the importance of views of life cannot be overemphasized.

In these circumstances, what seems to be still lacking is a consideration of ways to integrate promising ideas in various fields into a framework for a unified theory of information. Indeed this is too complex to be analyzed in detail here, but it will be helpful to define discussions and reconsider 
some philosophical suppositions that would be important also for other discussions toward a new information theory.

Therefore, we focus our argument on the relationship between systems theory and semiotics, or to put it more concretely, the relationship between autopoiesis theory and biosemiotics. The relationship between the two theories is relatively easy to consider because some arguments toward a new information theory have already been proposed in parallel based on these two theories. However, the most important point of the preference for systems theory and semiotics is that these theories give rise to two decisive viewpoints that seem potentially contradictory, so that the preference provokes a fruitful controversy which is conducive for the consideration of philosophical suppositions vital for a new information theory.

\section{The Relationship between Systems Theory and Semiotics on Cybersemiotics and Fundamental Informatics}

We will begin our argument by considering two frameworks that are both clearly based on systems theory and semiotics toward a new fundamental theory of information. One is the Cybersemiotics of Søren Brier and the other is Fundamental Informatics of Toru Nishigaki.

Brier regards the classical research program for information or communication science as 'information processing paradigm' and criticizes it (Brier, 1995, 1998, 2008). The information processing paradigm is a logical and mechanistic approach built on an objective information concept. He writes:

There are many arguments against this. Mine are from a biological perspective. I do not think that meaning can be fully represented in a syntactic logical form. Meaning is very much tied to biological existence (Brier, 1995, pp. 5-6).

Therefore, Brier insists that information processing paradigm needs to be replaced by a more comprehensive framework that includes such a biological perspective. What is then proposed is cybersemiotics as a new framework for a unified theory of information, in which he attempts to unite the two theoretical streams, cybernetics and semiotics, from a biological point of view (Brier, 1995, 1998, 2008).

On the other hand, Nishigaki develops his argument as Fundamental Informatics (FI) independently of Brier but with the similar intention to criticize the conventional approach to information and bridge the gap between natural sciences, in which aspects of the meaning of information are usually disregarded, and social sciences or the humanities, in which it is taken for granted that information is significant (Nishigaki, 2003, 2004). He also emphasizes that a biological perspective is indispensable for informatics, as can be seen in the following quotation:

These discussions about significance gradually made it clear that significance is inseparably related to biological phenomena. Significance means 'something that is important' and 'something that is valuable' to a living thing, and an information-bearing sign points to the existence of 'something that is valuable' (Nishigaki, 2003, pp.6-7 (p. 5 in English version)).

On the basis of this biological view of information, Nishigaki refers to systems theory and semiotics with the question "what is life?"

Before we turn to the discussion of the relationship between systems theory and semiotics on cybersemiotics and $\mathrm{FI}$, it will be useful to review the features of systems theory and semiotics separately in terms of their contribution to an information theory.

Note that, in this paper, the term 'systems theory' is mainly meant as second-order cybernetics originated by Heinz von Foerster and superimposed on the autopoiesis theory of Humberto Maturana and Francisco Varela. Second-order cybernetics is called 'cybernetics of cybernetics', which implies a focus shift from a system to an observing system. It regards a cognitive system or a living system as a self-organizing closed system; accordingly it is strongly associated with the radical turn of views of information from openness to closeness. To borrow Brier's phrase, "Information therefore is not something outside, but rather is a phenomenon created inside the organ- 
ism" (Brier, 1995, p. 7) ${ }^{1}$. From this point of view, we can see that information is not exchanged between systems, for each system is operationally closed.

Semiotics, on the other hand, contributes towards introducing aspects of meaning into an information theory. As is generally known, modern semiotics has two major paradigms, that is, a paradigm originating in the work of Charles Sanders Peirce and a paradigm proposed by Ferdinand de Saussure as a more general framework than just linguistics. The Saussurean stream of sign studies, usually called semiology, deals with mainly cultural or social aspects of signs, such as codes or structures typically found within human language. In contrast, Peircean semiotics is not strongly bound to cultural or social dimensions. It directs its attention mainly to each interpretation process of signs. From this point of view, we can say that Peircean semiotics is more applicable to informatics which focuses on a biological perspective, as it seems to have the possibility of expanding the purview of semiotics beyond human language and culture. In fact, there is a field called biosemiotics which was argued by Thomas A. Sebeok (Sebeok \& Umiker-Sebeok Eds., 1992) and has been developed by many researchers such as Jesper Hoffmeyer. Covering more fundamental semiotic phenomena of life, biosemiotics offers a view of the essential connection between life and meaning to a new information theory.

We will now return to our main subject, that is, the discussion of the relationship between systems theory and semiotics. From the review above, we can see that the idea of coexistence of life and cognition in second-order cybernetics and biosemiotics is the same. These two theories seem to be sharing the same standpoint that is expressed best by Brier when he states the following: "a bioconstructivism - that is, they see every living system constructing its own "life world"' (Brier, 2008 , p. 339). This leads to a constructive view of information, and that serves to overcome information processing paradigms in which objective information and its exchange and processing in the external given environment are presupposed.

Nevertheless, there is a potential contradiction between systems theory and semiotics, as the following section reveals. It can be for this reason that cybersemiotics necessarily approaches to semiotics, and $\mathrm{Fl}$, on the other hand, to systems theory, respectively.

The cybersemiotics of Brier can be primarily understood as a discussion that attempts to integrate systems theory and semiotics through the concept of 'sign games' that is something like Wittgenstein's concept of language games between prelanguage systems. The direct meeting point of the two theories in cybersemiotics is at the concept of 'eigenvalue' or 'eigenbehavior', which is used in von Foerster's discussion and means stable modes of system's dynamics when the system is perturbed repeatedly. That is to say, a sign is regarded as something like an eigenvalue established through communication, and its communicative meaning is regarded as something obtained or actualized through sign games.

Brier also argues that the concepts of eigenvalue and sign games fit Peirce's idea of habit into which semiosis (the semiotic process of the interpretation of signs composed of cooperation of three basic elements, a sign, its object and its interpretant) would develop. Although it might be felt to be confusing because an eigenvalue as a sign itself is generated by a sign process, we must understand that Peirce's semiotics is pan-semiotics, in other words, it regards all phenomena including natural processes as semiosis.

On the basis of such a pan-semiotic view, Brier eventually presents more comprehensive and metaphysical framework, which is a five-level ontological perspective that is derived from Peirce's pan-semiotics and modern science. The first level and the second level of that corresponds to Firstness and Secondness respectively, two of three categories proposed by Peirce, and the other three levels of Brier, which can be expressed by words such as quasi-semiotic, genuine semiotic and linguistic, would be included in the category of Thirdness of Peirce.

Cybersemiotics as a whole is now closer to semiotics than systems theory because of this ontological framework. The view of a sign as an eigenvalue recedes and the pan-semiotic scheme

\footnotetext{
${ }^{1}$ For the purposes of this paper, it is not necessary to discuss the definition of information in more detail here. However, its fundamental difficulty will be understood from the discussion in the third section of this paper.
} 
seems to be in the foreground. To quote Brier, "Sign making is thus immanent in nature, but manifest only in full triadic semiosis within living systems" (Brier, 2008, p. 390).

Compared with cybersemiotics, the FI of Nishigaki is closer to systems theory. He primarily uses autopoiesis theory to consider informational phenomena fundamentally connected with life, and uses semiotics mostly to reinforce views of life and views of meaning. Thus, he does not attempt to theoretically integrate the two theories, like Brier does.

Instead, FI fully approves of the constructive view of second-order cybernetics, which is directly related to radical constructivism. Radical constructivism is a radical approach to the problems of knowing proposed by Ernst von Glasersfeld, which has the assumption that "the thinking subject has no alternative but to construct what he or she knows on the basis of his or her own experience" (von Glasersfeld, 1995, p. 1). It deals with discussions about cognitive development such as constructing concepts without presupposing an objective external world, and as such is quite similar to the discussion on eigenvalue above.

It should also be noted that FI suggests an original view of transmission of information, which would be applicable to the discussion on signs as well, especially on their communicative aspects. Nishigaki writes:

A living thing is an essentially autonomous entity, but when it performs operations to transmit information and interpret its significance, are there any 'restrictions (restraints)' or 'bindings (constraints)' at work that affect its performance? Is not it precisely because a living system is restrained at least in some sense, that it is capable of transmitting information) (Nishigaki, 2003, p. 12 (p. 17 in English version))

Although it is intrinsically impossible to literally transmit information from the standpoint of systems theory, Nishigaki attempts to regard it as a 'fiction (pseudo-transmission)' and consider a way to understand the fiction based on systems theory.

It is from this point of view that he presents a new systemic concept, 'hierarchical autonomous system' or 'hierarchical autonomous communication system' (HACS), which enables us to consider such restrictions as the background of the 'fiction' of information transmission based on systems theory. We cannot discuss it in detail here, but HACS allows us to take a hierarchical view of plural autonomous systems by making much of a perspective of an observer, which may be changed strategically.

\section{A Potential Contradiction between Systems Theory and Semiotics}

It was pointed out in the previous section that each of cybersemiotics and FI shows affinity to semiotics and systems theory, respectively. That is, cybersemiotics eventually takes a pan-semiotic view; $\mathrm{FI}$, on the other hand, is mainly dependent on systems theory.

This implies the difficulty to construct a unified information theory by binding systems theory and semiotics. In this section, we will discuss this central issue. A good place to start is with an understanding of the treatment of the notion of information in autopoiesis theory. This offers the key to comprehending the potential problem between systems theory and semiotics.

An autopoietic system is defined as a system continuously regenerating the network of processes of production of components that produces the components (Maturana \& Varela, 1973/1980). One of the notable features of an autopoietic system, similar to a system as discussed by von Foerster, is that a system is closed by the recursive relation of production of components. Consequently, notions such as transmission of information and coding are also not admitted in autopoiesis theory: "Notions such as coding and transmission of information do not enter in the realization of a concrete autopoietic system because they do not refer to actual processes in it." (ibid. p. 90)

Varela suggests, however, that one of their original purposes was to propose a critical view, against the main stream in biology, on the use of such notions as transmission of information, and to regard information itself as unnecessary for understanding life (Varela, 1981, pp. 36-37). Their 
intention to refuse the notion of information for a system itself can be inferred from their original statements. The following quotation deserves careful attention in this respect.

An observer beholding an autopoietic system as a unity in a context that he also observes, and which he describes as its environment, may distinguish in it internally and externally generated perturbations, even though these are intrinsically indistinguishable for the autopoietic system itself. The observer can use these distinctions to make statements about the history of the autopoietic system which he observes, and he can use this history to describe an ambience (which he infers) as the domain in which the system exists. He cannot, however, infer from the observed correspondence between the ontogeny of the system and the ambience which this ontogeny describes, or from the environment in which he sees it, a constitutive representation of these in the organization of the autopoietic systems. The continuous correspondence between conduct and ambience revealed during ontogeny is the result of the homeostatic nature of the autopoietic organization, and not of the existence of any representation of the ambience in it; nor is it at all necessary that the autopoietic system should obtain or develop such a representation to persist in a changing ambience (Maturana \& Varela, 1973/1980, p. 99).

This remark is very important because it clearly shows that the standpoint of autopoiesis theory is fundamentally different from the standpoint of semiotics ${ }^{2}$. In autopoiesis theory, a system is operationally closed and all changes are internally determined, so that the system itself is considered unable to distinguish the origins of perturbations even if they are distinguishable for an observer. Contrary to this, it is presupposed in semiotics that the system or the subject itself distinguishes something such as the origins of perturbations, and such a presupposition seems even inevitable because the indispensable concept in semiotics is, needless to say, the concept of sign. In autopoiesis theory, it is presumed that the correspondence between system's conduct and its ambience is not the result of establishment of a representation of the ambience, and we cannot infer such a representation in the system from the observed phenomena. On the other hand, this understanding of the fields of such representations or signs is exactly what is attempted in semiotics.

This fundamental difference extracted from the discussion of autopoiesis theory reminds us of the general suspicion towards biosemiotics especially from mainstream biology: why do you relate life to signs? Although such a suspicion scarcely appears for anthropocentric semiotics, as subjects in semiotics change from humans to animals to bacteria or cells, it emerges as a problem contradictory to mechanical and materialistic views of life in natural-scientific biology.

The same thing may be said of autopoiesis theory. The fact that autopoiesis theory started as a theory of life to primarily explain the mechanism of a cell may account for its negative treatment of the notion of information. Autopoiesis theory indeed tends to emphasize its mechanical scheme and attempts to exclude not only the notion of information but also notions such as purpose, function and program. In this sense, autopoiesis theory is similar to the standpoint of natural sciences, even though it decisively differs from natural sciences in a certain sense.

This lack of relationship between natural sciences, in which we include autopoiesis theory, and biosemiotics reveals the great gap between mechanical views of life and semiotic views of life ${ }^{3}$. The former presumes that life just keeps operating and has no concern with the difference between inside and outside of itself, whereas the latter presumes that life distinguishes its environment as the other from itself and acts subjectively. This constitutes an aporia of views of life.

The importance of views of life cannot be overemphasized in arriving at a new information theory, as stated in the opening section of this article. We may say that some of the fundamental problems of a new information theory are concerned with a theory of life. Moreover, the remark above in autopoiesis theory that signs are unnecessary seems almost inevitable at least when a system is regarded as a closed system. The problem, therefore, cannot be solely in the relationship between

\footnotetext{
${ }^{2}$ The term 'semiotics' is used here to describe the study of sign in the general sense of the word.

${ }^{3}$ This is merely one remark in this context. The relationship between mainstream biology, autopoiesis theory and biosemiotics is complicated.
} 
autopoiesis theory and biosemiotics, but would be the most controversial point, which is hidden but emerges when we attempt to unify systems theory and semiotics for a new information theory.

\section{A Way to Overcome the Contradiction}

The problem considered above indicates the necessity of fundamental discussions to grasp the relationship between systems theory and semiotics and overcome the contradiction. To begin with, in order to gain a foothold for a discussion, let us take a look at the evaluation of semiotic notions, in other words informational notions, in autopoiesis theory from a different angle.

In autopoiesis theory, a system is regarded as a dual existence:

Systems as composite entities have a dual existence, namely, they exist as singularities that operate as simple unities in the domain in which they arise as totalities, and at the same time they exist as composite entities in the domain of the operation of their components (Maturana, 2002, p. 12).

That is, although a system is regarded as a singular entity in the domain of the interaction with its ambience, it is also regarded as a composite entity in the domain of the operation of its components.

Under this concept we can say that semiotic notions require a viewpoint of the former domain in addition to the latter, that is, they presuppose the interaction between a system as a unity and its ambience, while they presuppose the compositional operation toward establishment of such as a representation of the ambience. To put it briefly, semiotic notions consist of connecting these two domains.

The problem is the relationship of these two domains. Following the above, Maturana goes on to say: "The relation between these two domains is not causal; these two domains do not intersect, nor do the phenomena which pertain to one occur in the other" (ibid. p. 12).

This leads to the negative evaluation of semiotic notions. That is, semiotic notions are evaluated as merely views of an observer who arbitrarily relates these two domains, which have essentially no relations with each other. This is the reason why it is said that such a notion is "a cognitive notion which represents the interactions of the observer, not a phenomenon operative in the observed domain" (Maturana \& Varela, 1973/1980, p. 90). In order that autopoiesis theory may explain the living organization, it is required "to distinguish in it what pertains to the system as constitutive of its phenomenology from what pertains to our domain of description" (Maturana, 2002, p. 75).

Here, we have to remind ourselves of a feature of second-order cybernetics. We have pointed out earlier that second-order cybernetics implies a focus shift from a system to an observing system. From this point of view, second-order cybernetics itself can be seen as the work 'observing observing systems', and when we take into account the fact that we ourselves are also observing systems, it will be realized that the work of second-order cybernetics is involved in the dazzling recursiveness of observing which takes the form of observing observing observing...

Autopoiesis theory also shares this epistemological view by its very nature. An important point to emphasize in our context is that, as a consequence of this view, the relativity of observing or describing becomes clear:

No description of an absolute reality is possible. Such a description would require an interaction with the absolute to be described, but the representation which would arise from such an interaction would necessarily be determined by the autopoietic organization of the observer, not by the deforming agent; hence, the cognitive reality that it would generate would unavoidably be relative to the knower (Maturana, 2002, p. 121).

Maturana expresses this in brief as "Anything said is said by an observer".

As a result of this fundamental relativity of observation we need to reflect the above distinction between what pertains to the system as constitutive of its phenomenology and what pertains to our domain of description. Since any such distinction implies the work of an observer, it is impossible to strictly distinguish any systemic phenomenology from our description, nor can we insist that either 
explanation has an intrinsically superior status. In fact, Varela redefines his discussion in the same way and writes as follows:

When we say that teleonomic-symbolic explanations are not really necessary, it seems to me that we are succumbing to a prejudice of our historical tradition that it is time to revise, because in actual practice we cannot do without both operational and symbolic explanations. Our preference for causal explanations seems to be rooted in the understanding that 'causes' are 'out there' and reflects a state of affairs independent of the describer. This is, by the very argument used in AS, untenable (Varela, 1981, p. 44) .

Thus, we can say that applying autopoiesis theory to itself makes its own argument relativistic. This is also applicable to the negative evaluation of semiotic or informational notions in early autopoiesis theory.

In addition, Varela advances his view towards more comprehensive understanding of life and reevaluates notions such as information and purpose. He insists that we need to reduce our explanation to a symbolic explanation to cover the developmental or evolutional phenomena of life, even though he persists in saying that autopoietic understanding is "true on purely logical grounds" (Varela, 1981, p. 44).

This tentative positioning of semiotic notions in autopoiesis theory is still far from the actual discussions in biosemiotics that inquire of the connection between life and meaning. However, Evan Thompson who used to be Varela's co-worker says that Varela changed his mind in his later years; admitting the relationship between autopoiesis and the organism's sense-making, in other words teleology (Thompson, 2004, p. 391). Thompson interprets this as a feature of the coupling of an autopoietic system and its ambience (Thompson, 2004, p. 392).

We are unable to examine Thompson's discussion further because of space limitations, nor is it necessary to discuss his comments in detail. What matters is rather that we have already obtained a methodology to overcome the contradiction between systems theory and semiotics through some philosophical suppositions. We can now move onto examining those suppositions in more detail.

\section{Some Philosophical Suppositions Vital for a New Information Theory}

The previous discussion suggests some philosophical suppositions that would be important for a new information theory. Even if some of those suppositions seem self-evident, they are still worth highlighting because noting these important foundations is useful when we attempt to integrate various discussions into a new information theory.

Now, let us examine those philosophical suppositions. The following three points are to be discussed in the context of basic principles:

- Epistemology rather than ontology

- Constructivism rather than metaphysics

- Meta-theoretical recursiveness rather than linear consistency

Since the previous discussion was from the standpoint of systems theory, it might be considered that these three points are quite close to the features of second-order cybernetic systems theory. However, it is also noticeable that these points are features of some aspects of semiotics, as described later.

Although these three points can be defined differently and are furthermore intrinsically interrelated, we will consider them one by one.

\footnotetext{
${ }^{4}$ Varela used 'AS' to refer Maturana \& Varela 1973.
} 
First of all, we can say that epistemology is more important than ontology ${ }^{5}$ for a fundamental information theory. This would be a supposition inevitably implied in the shift from first-order cybernetics to second-order cybernetics, that is, the focus shift from a system to an observing system, as stated above.

The focus on epistemology is also shared with a part of biosemiotics. For instance, Jakob von Uexküll, who was a biologist reevaluated by Sebeok as a pioneer of biosemiotics, developed such an epistemological view in the famous theory of 'Umwelt' (Uexküll \& Kriszat, 1934/1970). He regarded animals as subjects, not merely objects, and proposed the concept of Umwelt as a surrounding world in which an animal perceives and acts as a subject. Umwelt is, to put it briefly, a real world for the particular animal. He argued that every subject including human beings lives in such a subjective world. It can even be said that space and time cannot exist without a subject. Therefore, the theory needs to be conscious of the standpoint of an observer. This is a good example of an epistemological approach similar to second-order cybernetics in the related fields of semiotics.

Looking back at the history of systems theory, Gregory Bateson's view of information is valuable to some extent, but it seems still insufficient in the sense of the shift from ontology to epistemology. Bateson's well-known definition of information, "a difference which makes a difference", regards every difference as potential information that appears as genuine information through life. However, to borrow Brier's phrase, Bateson's point of view has "the difficulty of determining to whom or what a difference makes a difference" (Brier, 2008, p. 179). In brief, it seems insufficient from an epistemological viewpoint.

Even so, it should be noted that Brier's cybersemiotic framework also seems somewhat similar to Bateson's worldview after all. Although this might be partly due to his dependence on Peirce's pan-semiotics, it is more due to the Brier's own view of 'reality' in the background. Brier seems to be struggling to understand reality or existence, as is demonstrated by the following quotations:

Although we have rightly abandoned the notion of 'objective reality' in second-order cybernetics, we should not give up the notion of a partly independent 'outside reality' (Brier, 2008, p. 93).

Maturana and Varela go too far when they claim that there is no world without an observer, that we live in a Multiverse created through our observing and acting, and that we can say nothing meaningful about the world as such (Brier, 2008, p. 194).

Consequently, he approaches an ontological pan-semiotic framework.

It must be noted, however, that we need to recognize that the renouncement of describing the absolute reality is essential in order to overcome the contradiction between systems theory and semiotics. The discussion in the previous section reveals the importance of shifting the question from the ontological one, which inquires into what kind of existence life itself is, to the epistemological one, which inquires into how we see life. What needs to be emphasized is that the ontological inquiry will eventually encounter the aporia of views of life. This is inexorably intertwined with the following point.

We can say that constructivism is more appropriate than metaphysics. A constructive view of cognition is expressed well by Maturana when he says, "Anything said is said by an observer". It is fundamentally different from a metaphysical worldview that assumes something independent of any observer.

Indeed we may be confused by the problem of how to think about reality or existence in constructive manner, but this feeling is considered to come from a confusion between "Most of the world is as it is whatever we think about it" and "There is something out there in addition to the world called 'the truth about the world'", as Richard Rorty implies from the standpoint of pragma-

\footnotetext{
${ }^{5}$ The word 'ontology' is used in the general or traditional sense of the word, that is, Heidegger's usage is not implied here.
} 
tism (Rorty, 1982, p. xxvi). There is no reason to doubt the former statement, but this does not mean that the latter is right.

We can consider, instead, that objectivistic concepts such as reality or existence are radically constructed by human beings. This is the very core of the discussion in von Glasersfeld's radical constructivism (von Glasersfeld, 1995). Uexküll would also seem to agree with this view because he regards the world as our Umwelt that is uniquely constructed by human beings.

Metaphysics seems to be required when we set up an ontological question. That is, it serves as a doctrine when we encounter a problem such as the aporia of views of life. Brier says, "One of the strengths of Peirce's semiotic philosophy is that qualia and mind - as semiosis - are installed in the metaphysics from the beginning." (Brier, 2008, p. 363).

Peirce's pan-semiotic metaphysics is actually useful in the sense that it eliminates the need to explain a path to a semiotic understanding of life. However, this strategy means simply excluding other views of life and abandoning meaningful discussions on their relationships. In addition, we have to say that the pan-semiotic worldview, in which everything is regarded as a (potential) sign, is close to Bateson's view of information, where every difference is regarded as potential information. As a result, the adoption of pan-semiotics yields some doubt about views of information, because the nearness to Bateson causes disregard of the radical turn of views of information from openness to closeness, which allows us to overcome the information processing paradigm.

However, we must add that Peirce's thought contains a kind of constructivism. As a matter of fact, Peirce describes a reality as something dependent on the ultimate decision of the community (Peirce, 5.316), which can be considered a constructive point of view. If this constructive view is recursively applied, Peircean semiotics will link more easily with epistemological constructivism than ontological metaphysics.

It follows from what has been said thus far that we require meta-theoretical recursiveness rather than linear consistency. If constructivism is radically considered, it is inevitable that the discussion takes on a meta-theoretical feature and implies a circularity of argument.

Maturana who emphasizes the role of an observer admits that even the observer itself arises in the conversations of observers:

The realization of autopoiesis, the living of the organism, as well as the observers themselves, all arise in the conversations of observers, so that all that there is, is languaging as coordinations of coordinations of consensual doings of observers that operate in language (Maturana, 2002, p. 32).

This is a good example to illustrate the meta-theoretical, recursive feature of the theory. We can recognize from this that the theory should not take the position to defend linear consistency but accept the recursiveness of the theory itself.

Consequently, we have to claim such a theory, to borrow von Glasersfeld's phrase, "to be no more than one possible model of thinking about the only world we can come to know, the world we construct as living subjects" (von Glasersfeld, 1995, p. 22). However, this is not to say that the discussion is arbitrary. What is important is that we can still examine its logical but recursive consistency and its experiential validity as a conceptual tool.

\section{Conclusion}

In this paper, we examined the relationship between systems theory and semiotics in order to discuss methodological issues related to a possible framework for a unified theory of information. Although we revealed that these two theories conflict with each other on their views of life, we also clarified that a way to overcome this contradiction can be found in systems theory itself. From this discussion, we derived three philosophical suppositions vital for a new information theory: epistemology rather than ontology, constructivism rather than metaphysics, meta-theoretical recursiveness rather than linear consistency. 
These three suppositions have some affinity to the features of second-order cybernetic systems theory. Likewise we can see similar features in certain aspects of semiotics, but we cannot say that modern semiotics is focusing on those aspects. Although there are a lot of practical analyses of meaning in semiotics, a careful examination of the philosophical bases for them seems to have been neglected.

The same thing may be said of biosemiotics. Biosemioticians are analyzing various aspects of life, but it seems that little attention has been paid to their philosophical bases. For example, the aporia of views of life, which was discussed in this paper, is considered to remain hidden and unsolved at the core of all biosemiotic questions.

Therefore, it seems reasonable to conclude that a mutually complementary relationship between systems theory and semiotics can be found based on these three suppositions. It can be developed further by systems theory and the actual analysis of meaning in semiotics. It calls for further investigation, but such investigation can be expected to lead to the construction of a new information theory.

\section{References}

Brier, S. (1995). Cyber-Semiotics: On Autopoiesis, Code-Duality and Sign Games in Bio-Semiotics. Cybernetics \& Human Knowing, 3(1), 3-14.

Brier, S. (1998). Cybersemiotics: A Transdisciplinary Framework for Information Studies. BioSystems, 46, 185-191.

Brier, S. (2008). Cybersemiotics: Why Information Is Not Enough!. Toronto: University of Toronto Press.

Glasersfeld, E. von (1995). Radical Constructivism: A Way of Knowing and Learning. London: Falmer Press.

Maturana, H. (2002). Autopoiesis, Structural Coupling and Cognition: A History of These and Other Notions in the Biology of Cognition, Cybernetics \& Human Knowing, 9(3/4), 5-34.

Maturana, H. and Varela, F. (1973). Autopoiesis: The Organization of the Living (Rpt. in Autopoiesis and Cognition: The Realization of the Living. D. Reidel, 1980).

Nishigaki, T. (2003). Ôtopoiêshisu ni motozuku Kiso Jôhô-gaku: Kaisô-gainen wo chûshin toshite (For the Establishment of Fundamental Informatics on the Basis of Autopoiesis: Consideration on the Concept of Hierarchical Autonomous Systems). SHISO, 951, 5-22. (English version available on http://www.digital-narcis.org/english/FI-English-01.pdf).

Nishigaki, T. (2004). Kiso Jôhô-gaku: Seimei kara Shakai e (Fundamental Informatics: From Life to Society). Tokyo: NTT Publishing.

Peirce, C. S. (1935). The Collected Papers of Charles Sanders Peirce: Vol.5. edited by Charles Hartshorne and Paul Weiss. Cambridge: Harvard University Press.

Rorty, R. (1982). Consequences of Pragmatism. Minneapolis: University of Minnesota Press.

Sebeok, T. A. and Umiker-Sebeok, J. (Eds.). (1992). Biosemiotics: The Semiotic Web 1991. Berlin: Mouton de Gruyter.

Thompson, E. (2004). Life and Mind: From Autopoiesis to Neurophenomenology. A Tribute to Francisco Varela. Phenomenology and the Cognitive Sciences 3, 381-398.

Uexküll, J. von and Kriszat, G. (1934). Streifzüge durch die Umwelten von Tieren und Menschen (Rpt. in S. Fischer Verlag, Frankfurt am Main, 1970).

Varela, F. J. (1981). Describing the Logic of the Living: The Adequacy and Limitations of the Idea of Autopoiesis. In M. Zeleny (Ed.). Autopoiesis: A Theory of the Living Organization (pp. 36-48). New York: Elsevier-North Holland.

\section{About the Author}

Yohei Nishida

is currently working toward a Ph.D. in the Graduate School of Interdisciplinary Information Studies (GSII) at the University of Tokyo. His research field is informatics and theory of life, including systems theory and biosemiotics. He is especially interested in the relationship between life and information. 